\title{
Infrared action spectroscopy of doubly charged PAHs and their contribution to the aromatic infrared bands
}

\author{
S. Banhatti ${ }^{1}$, J. Palotás ${ }^{2}$, P. Jusko ${ }^{3}$, B. Redlich ${ }^{2}$, J. Oomens ${ }^{2,4}$, S. Schlemmer ${ }^{1}$, and S. Brünken ${ }^{2}$ \\ ${ }^{1}$ I. Physikalisches Institut, Universität zu Köln, Zülpicher Str. 77, 50937 Köln, Germany \\ 2 Radboud University, Institute for Molecules and Materials, FELIX Laboratory, Toernooiveld 7, 6525ED Nijmegen, The Netherlands \\ e-mail: sandra.bruenken@ru.nl \\ ${ }^{3}$ Max Planck Institute for Extraterrestrial Physics, Gießenbachstraße 1, 85748 Garching, Germany \\ ${ }^{4}$ van 't Hoff Institute for Molecular Sciences, University of Amsterdam, Science Park 908, 1098XH Amsterdam, The Netherlands
}

Received 22 October 2020 / Accepted 14 February 2021

\begin{abstract}
The so-called aromatic infrared bands (AIBs) are attributed to emission of polycyclic aromatic hydrocarbons (PAHs). The observed variations toward different regions in space are believed to be caused by contributions of different classes of PAH molecules, that is to say with respect to their size, structure, and charge state. Laboratory spectra of members of these classes are needed to compare them to observations and to benchmark quantum-chemically computed spectra of these species. In this paper we present the experimental infrared (IR) spectra of three different PAH dications, naphthalene ${ }^{2+}$, anthracene ${ }^{2+}$, and phenanthrene ${ }^{2+}$, in the vibrational fingerprint region 500-1700 $\mathrm{cm}^{-1}$. The dications were produced by electron impact ionization of the vapours with $70 \mathrm{eV}$ electrons, and they remained stable against dissociation and Coulomb explosion. The vibrational spectra were obtained by IR predissociation of the $\mathrm{PAH}^{2+}$ complexed with neon in a 22-pole cryogenic ion trap setup coupled to a free-electron infrared laser at the Free-Electron Lasers for Infrared eXperiments (FELIX) Laboratory. We performed anharmonic density-functional theory calculations for both singly and doubly charged states of the three molecules. The experimental band positions showed excellent agreement with the calculated band positions of the singlet electronic ground state for all three doubly charged species, indicating its higher stability over the triplet state. The presence of several strong combination bands and additional weaker features in the recorded spectra, especially in the 10-15 $\mu \mathrm{m}$ region of the mid-IR spectrum, required anharmonic calculations to understand their effects on the total integrated intensity for the different charge states. These measurements, in tandem with theoretical calculations, will help in the identification of this specific class of doubly-charged PAHs as carriers of AIBs.
\end{abstract}

Key words. ISM: lines and bands - ISM: molecules - techniques: spectroscopic - methods: laboratory: molecular molecular data - line: identification

\section{Introduction}

The widespread mid-infrared (mid-IR) emission features observed in many astrophysical objects, such as HII regions, planetary nebulae (PNe), reflection nebulae, and young stellar objects, have been a topic of great interest since their detection in the 1970s and 1980s. Strong bands are observed at 3.3, 6.2, 7.7, 11.3, and $12.7 \mu \mathrm{m}$ along with several weak features, which together make up the unidentified infrared (UIR) or aromatic infrared bands (AIBs; Sellgren 1984; Sellgren et al. 1985; Jourdain de Muizon et al. 1990; Cohen et al. 1986). These bands are hypothesized to arise from a family of polycyclic aromatic hydrocarbons (PAHs) excited by absorption of ultraviolet (UV) radiation from nearby stars and their subsequent emission in the mid-IR region (Leger \& Puget 1984; Allamandola et al. 1989, 1985; Hudgins et al. 1997).

Depending on the conditions in the interstellar medium (ISM), different families of PAH molecules are proposed to exist, such as neutral and ionic variants of different size, shape, and hydrogenation states, all of which have an effect on the relative band intensities and band positions in the observed UIR bands (Hony et al. 2001; Dartois \& D'Hendecourt 1997). For example, the relative intensity variation between the 6.2, 7.7, 8.6, and $11.2 \mu \mathrm{m}$ bands has been attributed to the degree of ionization of PAHs (Galliano et al. 2008). Also, the $18.9 \mu \mathrm{m}$ band has been identified as a signature of multiply charged PAHs (Tielens 2008).

Identifying different classes of PAHs as carriers of UIR bands requires laboratory data of their gas-phase IR spectra. Several schemes have been used over the past decades to record the IR spectra of PAHs. In particular for the study of charged PAHs, the development of intense and widely tunable freeelectron lasers in facilities such as the Free-Electron Lasers for Infrared eXperiments (FELIX) Laboratory (Oepts et al. 1995) ${ }^{1}$ and $\mathrm{CLIO}^{2}$ has made it possible to implement sensitive action spectroscopic schemes such as infrared multiple photon dissociation (IRMPD). Gas-phase IR spectra of several small- to medium-sized cationic species have been recorded with the IRMPD scheme (e.g., naphthalene, phenanthrene, anthracene, coronene, and protonated naphthalene), pioneered by Oomens and coworkers (Oomens et al. 2000, 2001; Bakker et al. 2011; Lorenz et al. 2007). More recent works on the IR spectroscopy of gas phase PAH ions include larger species such as diindenoperylene $\left(\mathrm{C}_{32} \mathrm{H}_{16}{ }^{+}\right)$, dicoronylene $\left(\mathrm{C}_{48} \mathrm{H}_{20}{ }^{+}\right)$, hexaperi-hexabenzocoronene $\left(\mathrm{C}_{42} \mathrm{H}_{18}{ }^{+}\right)$(Zhen et al. 2017, 2018), and

\footnotetext{
1 https://www.ru.nl/felix/

2 http://old.clio.lcp.u-psud.fr/clio_eng/clio_eng.htm
} 
rubicene $\left(\mathrm{C}_{26} \mathrm{H}_{14}{ }^{+}\right)$(Bouwman et al. 2020) and PAH anions of naphthyl, anthracenyl, and pyrenyl (Gao et al. 2014). Their spectra have been shown to contain vibrational modes which could account for some of the features observed in the UIR bands. Pentagon-containing PAHs, for example, were shown to possess distinct vibrational modes at around $1100 \mathrm{~cm}^{-1}$ $(\sim 9.3 \mu \mathrm{m})$, and the ratio of the 9.3/6.2 $\mu \mathrm{m}$ band strength was used to estimate their relative abundance in several astronomical sources (Bouwman et al. 2020). Experimental results also verified that protonated nitrogen-containing species $\left(\mathrm{H}^{+} \mathrm{PANHs}\right)$ might indeed be responsible for the $6.2 \mu \mathrm{m}$ UIR emission (Alvaro Galué et al. 2010).

Another powerful action spectroscopic technique is infrared predissociation (IRPD), which requires cold ion conditions to tag the ions with a weakly bound rare-gas atom. Earlier IRPD studies applied to PAH ions used a molecular beam cooled by supersonic expansion, and they provided gas phase spectra of cold protonated (Ricks et al. 2009) and cationic (Piest et al. 1999) naphthalene and phenanthrene (Piest et al. 2001) via dissociation of their weakly bound complexes with Ar. With the advent of cryogenic ion trapping techniques, IRPD spectra can now be recorded at temperatures as low as $4 \mathrm{~K}$, allowing for the use of weaker bound rare-gas atoms such as $\mathrm{He}$ or $\mathrm{Ne}$ as tagging agents, introducing smaller shifts of vibrational bands in the experimental spectra (Asmis et al. 2002; Jašík et al. 2014; Günther et al. 2017; Gerlich et al. 2018; Jusko et al. 2019). The advantage of IRPD compared to multiphoton IRMPD spectroscopy is that dissociation happens after absorption of a single photon, with approximately constant efficiency, resulting in experimental vibrational spectra more closely resembling the linear absorption spectrum of the ions, both in intensity and band positions. In the past, we have successfully used this technique in our cryogenic ion trap instrument coupled to the widely tunable free electron lasers at the FELIX Laboratory to record narrowlinewidth spectra of PAH cations and related species (Jusko et al. 2018a,b; Panchagnula et al. 2020), and here we apply it for the spectroscopic characterization of PAH dications.

The presence of PAH dications and their formation in the ISM has been discussed previously by Leach (1986). Theoretical investigations on their stability and vibrational spectra have been carried out by Malloci et al. (2007a), Bakes et al. (2001a,b), and Bauschlicher \& Langhoff (1997). However, most of the laboratory work done so far in regards to the vibrational spectra of PAHs has been focused on their neutrals and monocations. There is no spectroscopic laboratory data on doubly charged PAHs in the IR regime except for an IRMPD study of hexa-perihexabenzocoronene, $\mathrm{C}_{42} \mathrm{H}_{18}{ }^{2+}$ (Zhen et al. 2017). In this work we present the IR predissociation spectra of the following three doubly charged PAHs using Ne-tagging in a cryogenic ion trap: naphthalene ${ }^{2+}\left(\right.$ naph $\left.^{2+}, \mathrm{C}_{10} \mathrm{H}_{8}{ }^{2+}\right)$, anthracene ${ }^{2+}\left(\right.$ anth $\left.^{2+}\right)$, and its isomer phenanthrene ${ }^{2+}\left(\right.$ phen $^{2+}$, both $\left.\mathrm{C}_{14} \mathrm{H}_{10}{ }^{2+}\right)$.

\section{Methods}

\subsection{Experimental methods}

The vibrational spectra of PAH dications were recorded using the FELion cryogenic 22-pole ion trap setup coupled to the free-electron laser FEL-2 at the FELIX Laboratory (Oepts et al. 1995). The FELion experimental setup has already been described in detail in Jusko et al. (2019). To provide a brief overview, the respective vapours of PAHs are ionized by electron impact ionization (EI), which results in the formation of several charged fragments including the doubly charged naphthalene cation $\left(\mathrm{C}_{10} \mathrm{H}_{8}{ }^{2+}\right.$, mass-to-charge ratio of $m / z=64$, naph ${ }^{2+}$ in the

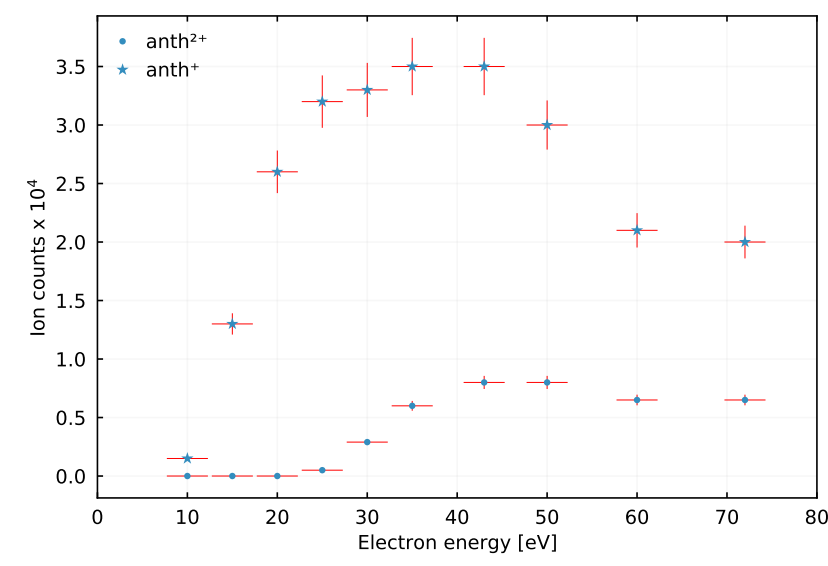

Fig. 1. Ionization yield for $m / z=89\left(\mathrm{anth}^{2+}\right)$ and $m / z=178\left(\mathrm{anth}^{+}\right)$ produced in a non-storage electron ionization source.

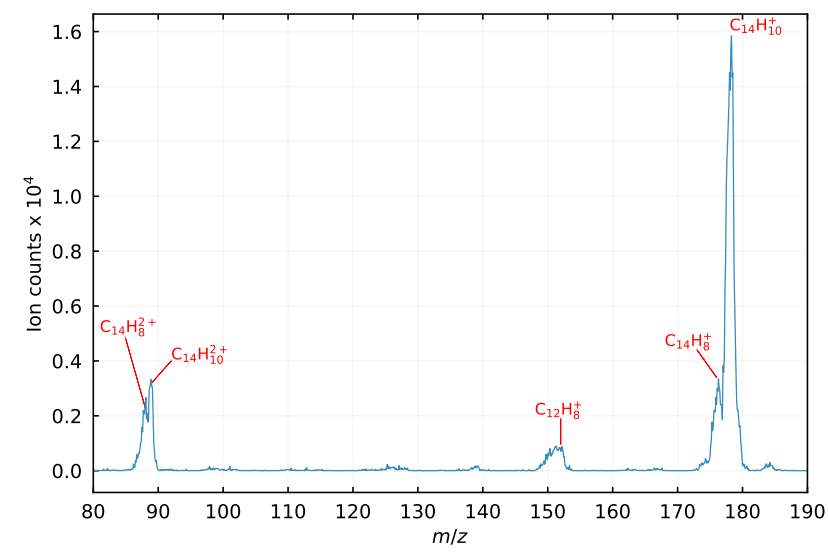

Fig. 2. Mass spectrum using phenanthrene precursor in the mass range $m / z=80-190$. The monocation at $m / z=178$, the doubly charged parent at $m / z=89$, and the $\mathrm{C}_{2} \mathrm{H}_{2}$ loss fragment at $m / z=152$ are shown in the figure.

following) and the doubly charged phenanthrene and anthracene cations $\left(\mathrm{C}_{14} \mathrm{H}_{10}{ }^{2+}, m / z=89\right.$, phen ${ }^{2+}$, and $a^{2+}{ }^{2+}$, resp. $)$. In our experiments, we used electron energies of $50-70 \mathrm{eV}$, which is well above the appearance energy of naph $^{2+}$ at $21.52 \mathrm{eV}$, and phen $^{2+}$ and anth ${ }^{2+}$ around $20 \mathrm{eV}$ (Holm et al. 2011; Malloci et al. 2007b; van der Burgt et al. 2018, 2019). The dication yield was found to be the highest around $50 \mathrm{eV}$ and remained constant at higher energies, as can be seen in Fig. 1, showing the anth ${ }^{2+}$ (and $\mathrm{anth}^{+}$) yield as a function of electron energy. Figure 2 shows a typical mass spectrum upon EI of phenanthrene vapor.

While naph ${ }^{2+}$ was produced efficiently with an RF storage source (Gerlich 1992), we could not produce high yields of phen $^{2+}$ or anth ${ }^{2+}$ ions in this way. This is probably due to the chemical quenching of initially produced dications by charge transfer and reactions with the neutral precursor and fragments in the high pressure $\left(10^{-5}\right.$ mbar $)$ storage source over the typical storage time of seconds. Hence, a non-storage EI source was used for these dications. The ions produced in the source were mass selected to within $m / z= \pm 2$ for the desired dication in a quadrupole mass filter. The RF of the quadrupole was tuned such that at least $80 \%$ of the ions transmitted belong to the dication $\mathrm{C}_{14} \mathrm{H}_{10}{ }^{2+}$ or $\mathrm{C}_{10} \mathrm{H}_{8}{ }^{2+}$ (see Fig. A.1). The mass selected ions were then complexed in situ with neon $(\mathrm{Ne})$ in a 22-pole cryogenic ion trap. Either pure $\mathrm{Ne}$ gas (in the case of anth ${ }^{2+}$ and phen $^{2+}$ ) or a 3:1 He:Ne gas mixture (in the case of naph ${ }^{2+}$ ) was pulsed into the ion trap for $100-150 \mathrm{~ms}$ at the beginning of the 
storage period, with the trap held at a temperature of $15 \mathrm{~K}$ or $6.3 \mathrm{~K}$, respectively. A second quadrupole mass filter with a mass resolution of better than $m / z=0.5$ was used to mass select the products formed in the ion trap, thus ensuring that only the target dications complexed with $\mathrm{Ne}$ were detected to record the spectrum.

For a doubly charged ion, the Ne-dication complex appears at a mass-to-charge ratio of $m / z=+10$ higher than the $\mathrm{PAH}^{2+}$ in the mass spectra, which was observed for all of the abovementioned ions; the tagging yield was of the order $30 \%$. In addition, we also observed doubly and triply tagged ions at $\mathrm{m} / \mathrm{z}=+20$ and +30 (see Fig. A.1). The Ne-dication complexes are stored in the trap for typically $2.6 \mathrm{~s}$, where they are irradiated by IR radiation from the free-electron laser FEL-2. The FEL was set to a narrow bandwidth (FWHM) of $0.4-0.5 \%$ at $10 \mathrm{~Hz}$ repetition rate and the spectrum was recorded in the range of $500-1800 \mathrm{~cm}^{-1}$ $(20-5.5 \mu \mathrm{m})$ with a typical laser pulse energy inside the trap of $10-40 \mathrm{~mJ}$. The IRPD spectra were recorded by measuring the depletion of $\mathrm{Ne}$-dication complex ion counts as a function of laser frequency. To account for fluctuations in the number of complex ions and varying laser energy during the course of a single scan, the spectra were normalized to laser pulse energy and baseline corrected before averaging over multiple scans. Saturation depletion measurements allowed us to decode the isomer ratios of molecular ions having two or more stable structures, see, for example, Jusko et al. (2018a). A detailed account of this technique is discussed in Jusko et al. (2019).

\subsection{Theoretical methods}

Geometry optimizations and frequency calculations were performed at the density functional theory (DFT) level. The Gaussian16 software package (Frisch et al. 2016) was used for all calculations as installed at the Cartesius supercomputer at SURFsara, Amsterdam. The vibrational frequencies of doubly charged PAHs were calculated within the harmonic approximation using the hybrid B3LYP functional with $6-311+G(d, p)$ basis set and scaled uniformly with 0.9679 (Andersson \& Uvdal 2005). Furthermore, anharmonic calculations within the vibrational second-order perturbation level of theory (VPT2) were performed as implemented in Gaussian 16 with the same functional and basis set combination. We should note here that the VPT2 method implemented in Gaussian 16 only provides a basic treatment of resonances. A more accurate calculation, which is beyond the scope of the present work, would require an explicit treatment of resonating polyads with variational approaches, as demonstrated earlier on the example of PAH molecules (Mackie et al. 2015, 2018; Chen 2018; Piccardo et al. 2015).

To investigate the effect of Ne-tagging on the vibrational spectra, additional harmonic calculations were performed on the naph $^{2+}-\mathrm{Ne}$ complexes. Several binding geometries of the $\mathrm{Ne}$ atom to the $\mathrm{PAH}$ are conceivable with the $\mathrm{Ne}$ atom on top of the molecular plane and in plane with the molecule. The five lowest energy conformers were optimized and harmonic vibrational frequencies were calculated (see Appendix B.1). Here the dispersion corrected wB97XD functional with a cc-pVTZ basis was used, which was found previously to work well for weakly-bound RG-ion complexes (Jusko et al. 2018a).

\section{Results and discussion}

\subsection{Naphthalene}

The experimental IRPD spectrum of Ne-tagged naph ${ }^{2+}(\mathrm{Ne}-$ IRPD) is shown in the top panel of Fig. 3. For comparison, the

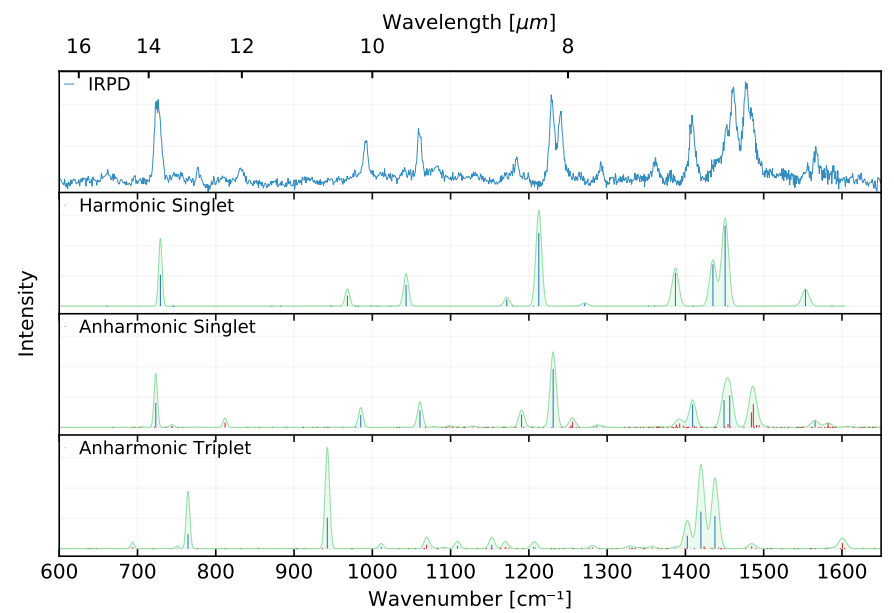

Fig. 3. IRPD spectrum of Ne-tagged naph ${ }^{2+}$ (top panel, blue) compared with calculated anharmonic and harmonic band positions of singlet $\mathrm{naph}^{2+}$ and anharmonic frequencies of triplet naph ${ }^{2+}$. Both fundamental modes (blue) and combination modes (red) are shown. The calculated spectrum was convoluted with a Gaussian lineshape function, where the width is given by the FEL bandwidth, and the area corresponds to the calculated intensity in $\mathrm{km} \mathrm{mol}^{-1}$.

calculated spectra of the singlet electronic ground state with ${ }^{1} A_{g}$ symmetry and of the $0.31 \mathrm{eV}$ higher lying triplet ${ }^{3} B_{3 g}$ electronic state are shown in the panels below. The peak positions from the fitted experimental spectrum and the corresponding band assignments based on the calculations are listed in Table 1. Most of the band positions in the Ne-IRPD spectrum can be readily assigned to the singlet state of naph ${ }^{2+}$, and they are in excellent agreement with the calculated anharmonic frequencies to within $10 \mathrm{~cm}^{-1}$ for the fundamental bands.

To account for the influence of the Ne-tag on the vibrational band positions, we compared harmonic DFT calculations for bare and different conformers of Ne-tagged naph ${ }^{2+}$. They revealed negligible band shifts of $<1 \mathrm{~cm}^{-1}$ for most of the bands, with only a few bands maximally shifted by $5 \mathrm{~cm}^{-1}$ (see Appendix B.1). The IRPD spectrum of the Ne-tagged species can therefore be viewed as an excellent proxy for that of the bare ion.

Only three of the experimental bands, at $1361 \mathrm{~cm}^{-1}$, $1240 \mathrm{~cm}^{-1}$, and $777 \mathrm{~cm}^{-1}$, could not be assigned in a straightforward manner. The band at $1362 \mathrm{~cm}^{-1}$ lies close to weak combination bands predicted at 1361 and $1365 \mathrm{~cm}^{-1}$ (not listed in Table 1). Several strong predicted combination bands were assigned to a weak experimental feature at $1381 \mathrm{~cm}^{-1}$, and they could also be assigned to the $1362 \mathrm{~cm}^{-1}$ band if we assume large anharmonic shifts unaccounted for by the calculations. Another feature at $832 \mathrm{~cm}^{-1}$ assigned to a predicted band at $812 \mathrm{~cm}^{-1}$ shows a similarly large deviation. For combination bands, larger deviations of calculated anharmonic band positions are expected when using VPT2, so we tentatively assigned these features to one or a combination of the above combination bands.

The rather strong feature at $1240 \mathrm{~cm}^{-1}$ is close to the strong $\mathrm{C}-\mathrm{H}$ in plane bending vibrational mode at $1229 \mathrm{~cm}^{-1}$, which almost appears as a doublet. From the calculations, we can exclude that the symmetry breaking induced by the Ne-tag has a significant influence on the spectrum, and that the presence of multiple $\mathrm{Ne}$-ion isomers are responsible for the observed splitting of the band. We therefore assume that the blue-shifted feature at $1240 \mathrm{~cm}^{-1}$ is due to a combination band of the strong $\mathrm{C}-\mathrm{H}$ bending mode with a vibration involving only the 
Table 1. Experimentally measured band positions $v_{\text {vib }}\left(\mathrm{cm}^{-1}\right)$, FWHM $\delta\left(\mathrm{cm}^{-1}\right)$, and relative intensities of the naphthalene dication compared to DFT computed harmonic (scaled by 0.9679 ) and anharmonic fundamental and combination mode positions and intensities $I\left(\mathrm{kmmol}^{-1}\right)>$ $5 \mathrm{~km} \mathrm{~mol}^{-1}$.

\begin{tabular}{|c|c|c|c|c|c|c|c|c|c|}
\hline \multicolumn{3}{|c|}{ IRPD (this work) } & \multicolumn{3}{|c|}{ Anharmonic calculation } & \multicolumn{3}{|c|}{ Harmonic calculation } & \multirow[t]{2}{*}{ Symmetry } \\
\hline$v_{\text {vib }}$ & $\delta$ & $I_{\text {rel }}$ & $v_{\text {vib }}$ & $I$ & $I_{\text {rel }}$ & $v_{\text {vib }}$ & $I$ & $I_{\text {rel }}$ & \\
\hline & & & 589 & 10.1 & 0.05 & 577 & 7.7 & 0.03 & $\mathrm{~b}_{2 u}$ \\
\hline $726(1)$ & 9.1 & 0.96 & 723 & 77.2 & 0.42 & 729 & 98.6 & 0.39 & $\mathrm{~b}_{3 u}$ \\
\hline $777(1)^{(a)}$ & 3.9 & 0.16 & & & & & & & \\
\hline $832(1)$ & 6 & 0.12 & $812^{(b)}$ & 15 & 0.08 & & & & $\mathrm{~b}_{2 u}$ \\
\hline $992(1)$ & 7.7 & 0.45 & 985 & 38.9 & 0.21 & 968 & 33.3 & 0.13 & $\mathrm{~b}_{2 u}$ \\
\hline $1060(1)$ & 6.8 & 0.57 & 1061 & 54.1 & 0.29 & 1043 & 67.3 & 0.27 & $\mathrm{~b}_{1 u}$ \\
\hline 1184(1) & 7.7 & 0.22 & 1191 & 41 & 0.22 & 1172 & 21 & 0.08 & $\mathrm{~b}_{2 u}$ \\
\hline $1229(1)$ & 7.3 & 0.96 & 1231 & 184 & 1.00 & 1213 & 231.1 & 0.91 & $\mathrm{~b}_{2 u}$ \\
\hline $1240(1)$ & 7.8 & 0.78 & & & & & & & \\
\hline \multirow[t]{2}{*}{$1266^{(c)}$} & & & $1254^{(b)}$ & 6.6 & 0.04 & & & & $\mathrm{~b}_{2 u}$ \\
\hline & & & $1256^{(b)}$ & 18.1 & 0.10 & & & & $\mathrm{~b}_{2 u}$ \\
\hline $1292(1)$ & 4.9 & 0.16 & 1287 & 6.1 & 0.03 & 1271 & 8.5 & 0.03 & $\mathrm{~b}_{1 u}$ \\
\hline $1362(1)$ & 7.8 & 0.20 & - & - & - & & & & \\
\hline \multirow[t]{2}{*}{$1381^{(c)}$} & & & $1388^{(b)}$ & 8.9 & 0.05 & & & & $\mathrm{~b}_{1 u}$ \\
\hline & & & $1393^{(b)}$ & 12.4 & 0.07 & & & & $\mathrm{~b}_{1 u}$ \\
\hline- & - & - & $1397^{(b)}$ & 5.5 & 0.03 & & & & $\mathrm{~b}_{1 u}$ \\
\hline $1409(1)$ & 8.9 & 0.67 & 1409 & 72.5 & 0.39 & 1387 & 104.6 & 0.41 & $\mathrm{~b}_{1 u}$ \\
\hline \multirow[t]{2}{*}{$1453(2)$} & 21.5 & 0.49 & 1449 & 86.3 & 0.47 & 1435 & 131.7 & 0.52 & $\mathrm{~b}_{1 u}$ \\
\hline & & & $1454^{(b)}$ & 10.6 & 0.06 & & & & $\mathrm{~b}_{2 u}$ \\
\hline 1461(1) & 7.2 & 0.73 & 1457 & 101.1 & 0.55 & 1451 & 253.7 & 1.00 & $\mathrm{~b}_{2 u}$ \\
\hline \multirow[t]{4}{*}{$1480(1)$} & 17.6 & 1.00 & $1484^{(b)}$ & 47.4 & 0.26 & & & & $\mathrm{~b}_{2 u}$ \\
\hline & & & $1487^{(b)}$ & 73.1 & 0.40 & & & & $\mathrm{~b}_{2 u}$ \\
\hline & & & $1491^{(b)}$ & 7.5 & 0.04 & & & & $\mathrm{~b}_{1 u}$ \\
\hline & & & $1494^{(b)}$ & 6.9 & 0.04 & & & & $\mathrm{~b}_{2 u}$ \\
\hline $1567(1)$ & 21.2 & 0.22 & 1566 & 21.1 & 0.11 & 1553 & 51.8 & 0.20 & $\mathrm{~b}_{2 u}$ \\
\hline- & - & - & $1582^{(b)}$ & 13.9 & 0.14 & & & & $\mathrm{~b}_{2 u}$ \\
\hline
\end{tabular}

Notes. Values in brackets denote uncertainties $(1 \sigma)$ in units of the last significant digit. ${ }^{(a)}$ See text for discussion on this band. ${ }^{(b)}$ Combination bands for singlet naph ${ }^{2+} .{ }^{(c)}$ These bands are either too weak or broad due to closely located bands and cannot be fitted.

Ne-tag, a phenomenon often seen in rare-gas tagging experiments (Brünken et al. 2019). The lowest harmonic fundamental frequencies involving the Ne-atom are shown in Fig. B.1. They fall in the $5-60 \mathrm{~cm}^{-1}$ wavenumber range, and they are thus lying close to the observed difference between the two experimental features. However, we should note that our calculations also show a Fermi resonance of the $1229 \mathrm{~cm}^{-1}$ mode with a closelying combination band of the same symmetry, which might gain intensity due to this interaction.

The weak band at $777 \mathrm{~cm}^{-1}$ appears to be coincident with a predicted fundamental of triplet naph ${ }^{2+}$ at $764 \mathrm{~cm}^{-1}$, but the absence of a much stronger predicted triplet mode around $943 \mathrm{~cm}^{-1}$ in the experimental spectrum suggests otherwise. To test the possible presence of the electronically excited triplet state, we performed saturation depletion measurements on several strong bands assigned to singlet naph ${ }^{2+}$, revealing an abundance of at least $80 \%$ of the ground electronic state. This indicates that up to $20 \%$ of the formed dications could be in the electronically excited triplet state or a different isomer (Leach et al. 1989a; Solano \& Mayer 2015). Since we do not observe any other triplet bands as expected from the calculated spectrum in Fig. 3 (bottom panel), the latter is more likely.

Whereas the calculated, scaled harmonic band positions of the singlet state correlate well with those from the anharmonic calculation, we can observe large shifts (up to $20 \mathrm{~cm}^{-1}$ ) between both levels of theory, indicating the need to include mode dependent anharmonic effects to correctly describe the vibrational PAH dication spectra. Furthermore, the strong combination bands between 1480 and $1490 \mathrm{~cm}^{-1}$ observed in the experimental spectrum can be accounted for only with anharmonic calculations. The narrow linewidths observed in our Ne-IRPD spectrum can serve as a benchmark for anharmonic calculations of PAH dications just as previously shown for PAH neutrals (Mackie et al. 2015; Maltseva et al. 2015).

\subsection{Phenanthrene}

The IRPD spectrum of phen ${ }^{2+}-\mathrm{Ne}$ compares well with the calculated anharmonic spectrum of the ${ }^{1} A_{1}$ singlet electronic state as seen in Fig. 4. Table 2 shows the peak positions with the assigned anharmonic and harmonic frequencies with many of the peak positions coinciding within $10 \mathrm{~cm}^{-1}$ of the calculated anharmonic spectrum for the singlet ground state. Again, we do not observe any bands that could be assigned to the triplet ${ }^{3} B_{2}$ electronic state $0.44 \mathrm{eV}$ higher (Table C.1).

The bands below $800 \mathrm{~cm}^{-1}$ show larger shifts $\left(<20 \mathrm{~cm}^{-1}\right)$ likely due to larger anharmonic effects. Several combination bands can be observed, most of which are weak and overshadowed by the strong fundamental bands, except for the three isolated features at 1000, 1246, and $1355 \mathrm{~cm}^{-1}$. Another strong feature at $1427 \mathrm{~cm}^{-1}$ is a mix of fundamental and combination bands making the overall feature appear broad. 


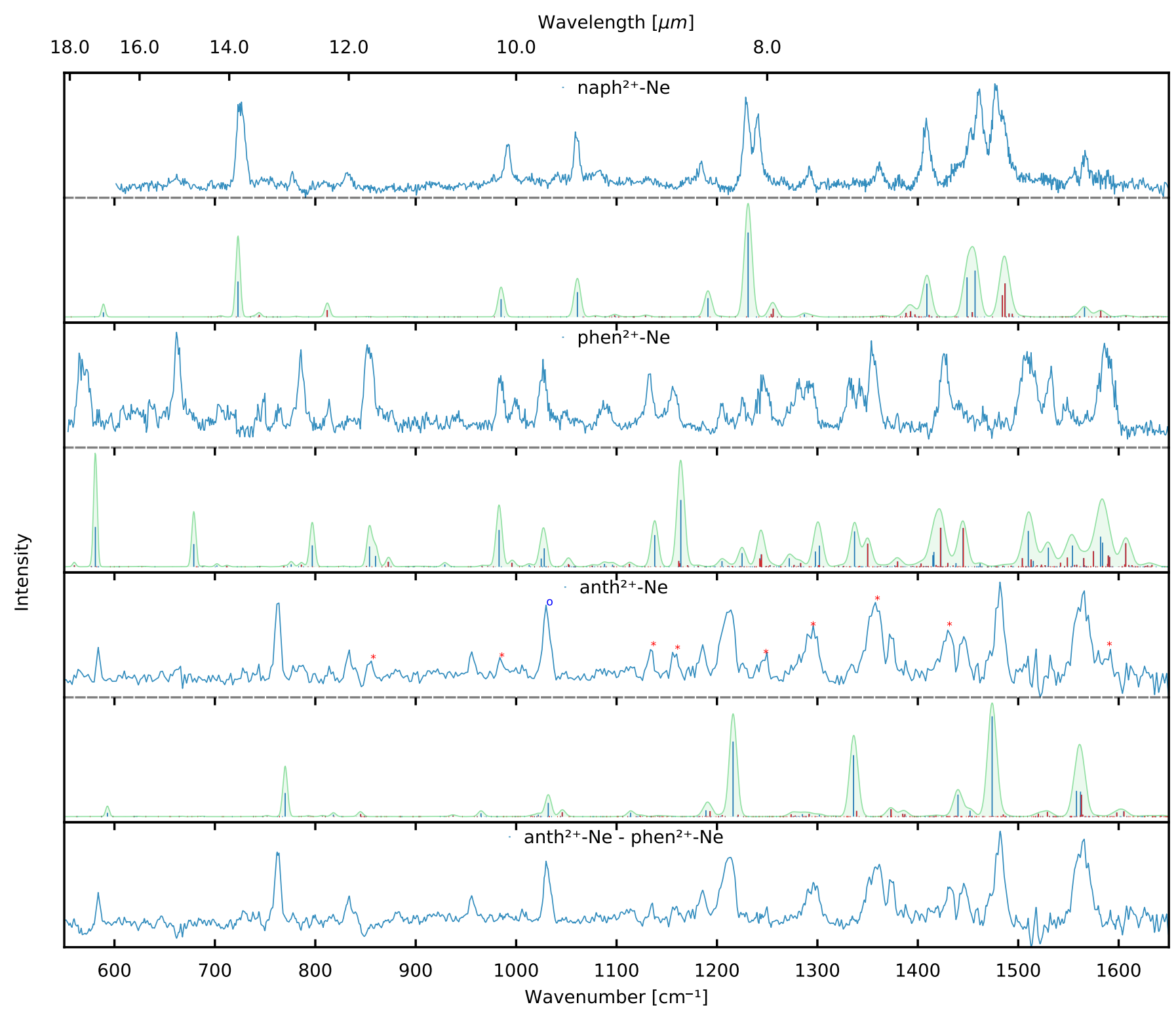

Fig. 4. IRPD spectrum of Ne-tagged dications naph ${ }^{2+}$, phen $^{2+}$, and anth ${ }^{2+}$ compared to calculated anharmonic (B3LYP/6-311+G(d,p)) spectra, respectively. The calculated spectrum was convoluted with a Gaussian lineshape function, where the width is given by the FEL bandwidth, and the area corresponds to the calculated intensity in $\mathrm{km} \mathrm{mol}^{-1}$. The spectrum in the bottom panel is the anth ${ }^{2+}-\mathrm{Ne}$ spectrum subtracted by $30 \%$ of the weight of the relative intensity of the phen ${ }^{2+}-\mathrm{Ne}$ spectrum to account for the contaminated bands.

We observe a similar doublet feature in the phen ${ }^{2+}$ spectrum at 1333 and $1343 \mathrm{~cm}^{-1}$. As discussed above for naph ${ }^{2+}$, we suspect this blue-shifted feature to be due to a strong combination band involving the Ne-tag. However, similar to naph ${ }^{2+}$, our calculations reveal a Fermi resonance of the fundamental predicted at $1337 \mathrm{~cm}^{-1}$ with two combination modes at 1350 and $1378 \mathrm{~cm}^{-1}$.

\subsection{Anthracene}

The lower panels in Fig. 4 show the Ne-IRPD spectrum of anth ${ }^{2+}$ compared to calculated anharmonic spectra of its singlet ${ }^{1} A_{g}$ electronic state. The band assignments are shown in Table 3. Due to the higher symmetry $\left(\mathrm{D}_{2 h}\right)$ of anth ${ }^{2+}$ compared to phen ${ }^{2+}$ $\left(C_{2 v}\right)$, the spectrum shows fewer and less intense vibrational bands. Once again, we do not see any trace of the energetically higher-lying $(0.79 \mathrm{eV})$ triplet ${ }^{3} B_{3 g}$ electronic state (Table C.1).
Several bands in the experimental spectrum coincide with the phen ${ }^{2+}$ bands (see caption in Fig. 4). These phen ${ }^{2+}$ bands appear to be due to contamination from the previous experiment where phenanthrene was introduced in the source. The $1357 \mathrm{~cm}^{-1}$ band, for example, is a convolution of three bands, two of which are assigned to anth ${ }^{2+}$ with some contribution from phen $^{2+}$ contamination of its $1355 \mathrm{~cm}^{-1}$ combination band.

Since phen ${ }^{2+}$ is the more stable isomer based on our calculations (by $0.7 \mathrm{eV}$ ), it is possible that the phen ${ }^{2+}$ bands observed in the anth ${ }^{2+}$ spectrum are due to isomerization of anth ${ }^{2+}$ to $\mathrm{phen}^{2+}$ during the electron impact ionization process, as has been predicted theoretically for the anthracene monocations (Johansson et al. 2011). To verify this, we also recorded spectra at lower ionization energy $(30 \mathrm{eV})$ expecting a change in the population of the phen ${ }^{2+}$ isomer and consequently a change in the peak intensity of phen ${ }^{2+}$ bands relative to the anth ${ }^{2+}$ bands. This method was successfully implemented for benzylium and 
Table 2. Experimentally measured band positions $v_{\text {vib }}\left(\mathrm{cm}^{-1}\right)$, FWHM $\sigma\left(\mathrm{cm}^{-1}\right)$, and relative intensities of the phenanthrene dication compared to DFT computed harmonic (scaled by 0.9679 ) and anharmonic fundamental and combination mode positions and intensities $I\left(\mathrm{~km} \mathrm{~mol}^{-1}\right)>$ $5 \mathrm{~km} \mathrm{~mol}^{-1}$.

\begin{tabular}{|c|c|c|c|c|c|c|c|c|}
\hline \multicolumn{3}{|c|}{ IRPD (this work) } & \multicolumn{3}{|c|}{ Anharmonic calculation } & \multicolumn{3}{|c|}{ Harmonic calculation } \\
\hline$v_{\text {vib }}$ & $\sigma$ & $I_{\text {rel }}$ & $v_{\mathrm{vib}}$ & $I$ & $I_{\text {rel }}$ & $v_{\mathrm{vib}}$ & $I$ & $I_{\text {rel }}$ \\
\hline $569(1)$ & 13 & 0.81 & 581 & 78.7 & 0.60 & 569 & 81.3 & 0.34 \\
\hline $663(1)$ & 9 & 1 & 679 & 44.9 & 0.34 & 645 & 34.9 & 0.15 \\
\hline $786(1)$ & 9 & 0.77 & 797 & 42.3 & 0.32 & 772 & 52.3 & 0.22 \\
\hline \multirow[t]{2}{*}{$854(1)$} & 12 & 0.98 & 854 & 40.1 & 0.30 & 836 & 40.3 & 0.17 \\
\hline & & & 860 & 21.4 & 0.16 & 848 & 35.2 & 0.15 \\
\hline $875^{(b)}$ & & & $873^{(a)}$ & 9.8 & 0.07 & & & \\
\hline $985(1)$ & 9 & 0.58 & 983 & 72.7 & 0.55 & 962 & 83.5 & 0.35 \\
\hline $1000(1)$ & 12 & 0.28 & $996^{(a)}$ & 7.8 & 0.06 & & & \\
\hline \multirow[t]{2}{*}{$1027(1)$} & 10 & 0.65 & 1025 & 16.4 & 0.12 & 1008 & 22.9 & 0.10 \\
\hline & & & 1028 & 36.5 & 0.28 & 1010 & 39.6 & 0.17 \\
\hline- & - & - & $1052^{(a)}$ & 5.1 & 0.04 & & & \\
\hline $1088(1)$ & 14 & 0.28 & 1088 & 5.8 & 0.04 & 1069 & 4 & 0.02 \\
\hline- & - & - & $1113^{(a)}$ & 5.8 & 0.04 & & & \\
\hline 1133(1) & 11 & 0.56 & 1138 & 62.6 & 0.47 & 1120 & 72.9 & 0.31 \\
\hline \multirow[t]{3}{*}{$1156(1)$} & 13 & 0.44 & $1162^{(a)}$ & 11.4 & 0.09 & & & \\
\hline & & & $1163^{(a)}$ & 6.7 & 0.05 & & & \\
\hline & & & 1164 & 131.9 & 1.00 & 1144 & 154.3 & 0.65 \\
\hline $1205(1)$ & 7 & 0.28 & 1205 & 11.2 & 0.08 & 1184 & 15.2 & 0.06 \\
\hline $1226(1)$ & 9 & 0.3 & 1225 & 27 & 0.20 & 1216 & 82.5 & 0.35 \\
\hline \multirow[t]{3}{*}{$1246(1)$} & 14 & 0.53 & $1243^{(a)}$ & 16.6 & 0.13 & & & \\
\hline & & & $1244^{(a)}$ & 12.9 & 0.10 & & & \\
\hline & & & $1244^{(a)}$ & 24.5 & 0.19 & & & \\
\hline \multirow[t]{2}{*}{$1283(2)$} & 23 & 0.47 & 1272 & 16.9 & 0.13 & 1255 & 29.3 & 0.12 \\
\hline & & & $1283^{(a)}$ & 7.4 & 0.06 & & & \\
\hline \multirow[t]{2}{*}{ 1294(1) } & 8 & 0.49 & 1298 & 30.2 & 0.23 & 1279 & 31.5 & 0.13 \\
\hline & & & 1302 & 41.8 & 0.32 & 1282 & 36.1 & 0.15 \\
\hline 1333(1) & 11 & 0.53 & $1337^{(c)}$ & 69.9 & 0.53 & 1330 & 235.9 & 1.00 \\
\hline 1343(1) & 6 & 0.47 & $1350^{(a),(c)}$ & 45.9 & 0.35 & & & \\
\hline \multirow[t]{3}{*}{$1355(1)$} & 11 & 0.91 & $1377^{(a),(c)}$ & 2.7 & 0.02 & & & \\
\hline & & & 1378 & 0.6 & 0.00 & & & \\
\hline & & & $1380^{(a)}$ & 10.5 & 0.08 & & & \\
\hline - & - & - & $1403^{(a)}$ & 6.4 & 0.05 & & & \\
\hline \multirow[t]{4}{*}{$1427(1)$} & 13 & 0.79 & 1415 & 23 & 0.17 & 1397 & 4.1 & 0.02 \\
\hline & & & 1416 & 29.3 & 0.22 & 1399 & 164.9 & 0.70 \\
\hline & & & $1423^{(a)}$ & 77 & 0.58 & & & \\
\hline & & & $1430^{(a)}$ & 7.5 & 0.06 & & & \\
\hline \multirow[t]{2}{*}{$1439^{(b)}$} & & & 1438 & 7.3 & 0.06 & 1419 & 9.2 & 0.04 \\
\hline & & & $1445^{(a)}$ & 76.5 & 0.58 & & & \\
\hline- & - & - & 1462 & 6.8 & 0.05 & 1448 & 3.1 & 0.01 \\
\hline \multirow[t]{4}{*}{$1510(1)$} & 18 & 0.86 & 1510 & 70.8 & 0.54 & 1493 & 132.3 & 0.56 \\
\hline & & & $1504^{(a)}$ & 17 & 0.13 & & & \\
\hline & & & $1513^{(a)}$ & 14.4 & 0.11 & & & \\
\hline & & & 1515 & 11.7 & 0.09 & 1500 & 22.9 & 0.10 \\
\hline 1532(1) & 7 & 0.65 & 1530 & 37.8 & 0.29 & 1519 & 82.3 & 0.35 \\
\hline & - & - & $1542^{(a)}$ & 8.2 & 0.06 & & & \\
\hline \multirow{3}{*}{ 1549(4) } & 15 & 0.23 & 1554 & 42 & 0.32 & 1540 & 25.2 & 0.11 \\
\hline & & & $1549^{(a)}$ & 18.3 & 0.14 & & & \\
\hline & & & $1565^{(a)}$ & 17.2 & 0.13 & & & \\
\hline \multirow[t]{7}{*}{$1588(1)$} & 15 & 0.93 & $1575^{(a)}$ & 30.8 & 0.23 & & & \\
\hline & & & 1582 & 59.5 & 0.45 & 1565 & 200.9 & 0.85 \\
\hline & & & 1584 & 47.7 & 0.36 & 1582 & 100.3 & 0.43 \\
\hline & & & $1589^{(a)}$ & 5.8 & 0.04 & & & \\
\hline & & & $1590^{(a)}$ & 22.2 & 0.17 & & & \\
\hline & & & $1591^{(a)}$ & 19.2 & 0.15 & & & \\
\hline & - & - & $1607^{(a)}$ & 46.5 & 0.35 & & & \\
\hline
\end{tabular}

Notes. Values in brackets denote uncertainties $(1 \sigma)$ in units of the last significant digit. ${ }^{(a)}$ Combination bands. ${ }^{(b)}$ These bands are either too weak or broad due to closely located bands and cannot be fitted. ${ }^{(c)}$ See text for discussion on this band.
Table 3. Experimentally measured band positions $v_{\text {vib }}\left(\mathrm{cm}^{-1}\right)$, FWHM $\sigma\left(\mathrm{cm}^{-1}\right)$ and relative intensities of the anthracene dication compared to DFT computed harmonic (scaled by 0.9679 ) and anharmonic fundamental and combination mode positions and intensities $I\left(\mathrm{~km} \mathrm{~mol}^{-1}\right)>$ $5 \mathrm{~km} \mathrm{~mol}^{-1}$.

\begin{tabular}{|c|c|c|c|c|c|c|c|c|}
\hline \multicolumn{3}{|c|}{ IRPD (this work) } & \multicolumn{6}{|c|}{ Anharmonic calculation Harmonic calculation } \\
\hline$v_{\mathrm{vib}}$ & $\sigma$ & $I_{\text {rel }}$ & $v_{\text {vib }}$ & $I$ & $I_{\text {rel }}$ & $v_{\text {vib }}$ & $I$ & $I_{\text {rel }}$ \\
\hline $584(1)$ & 3.5 & 42 & 593 & 13 & 0.038 & 580 & 12.1 & 0.029 \\
\hline $763(1)$ & 6.3 & & 770 & 80.9 & 0.235 & 757 & 90.9 & 219 \\
\hline $834(1)$ & 7.1 & 0.29 & 818 & 6.7 & 019 & 804 & 12.4 & 0.030 \\
\hline- & - & - & $845^{(a)}$ & 8.6 & 0.025 & & & \\
\hline $855(1)^{(b)}$ & 6.6 & 0.19 & - & - & - & - & - & \\
\hline $956(1)$ & 7.7 & 0.29 & 965 & 11.4 & 0.033 & 944 & 13.2 & 0.032 \\
\hline $985(3)^{(b)}$ & 9.9 & 0.2 & - & - & - & - & - & \\
\hline- & - & . & 1022 & 6.7 & 0.019 & 992 & 6.8 & 0.016 \\
\hline 1030(1) & 8.8 & 0.82 & 103 & 47.1 & 0.137 & 1013 & 60.7 & 0.147 \\
\hline - & - & - & $1046^{(a)}$ & 15 & 0.044 & & & \\
\hline- & - & - & 1114 & 13.4 & 0.039 & 1093 & 21.5 & 0.052 \\
\hline $1134(1)^{(b)}$ & 7.6 & 0.33 & - & - & - & - & - & \\
\hline $1158(1)^{(b)}$ & 9.2 & 0.29 & - & - & - & - & - & \\
\hline \multirow[t]{2}{*}{$1185(1)$} & 9.3 & 0.35 & 1189 & 21.7 & 0.063 & 1166 & 33.2 & 0.080 \\
\hline & & & $1193^{(a)}$ & 18.8 & 0.055 & & & \\
\hline \multirow[t]{3}{*}{ 1211(1) } & 16 & 0.81 & $1205^{(a)}$ & 5.1 & 0.015 & & & \\
\hline & & & 1216 & 257.4 & 0.748 & 1195 & 292.1 & 0.70 \\
\hline & & & $1221^{(a)}$ & 6.1 & 0.018 & & & \\
\hline $1246(1)^{(b)}$ & 11.3 & 0.24 & - & - & - & - & - & \\
\hline - & - & - & $1274^{(a)}$ & 8 & 0.023 & & & \\
\hline - & - & - & 1285 & 8.6 & 0.025 & 1263 & 28.1 & 0.06 \\
\hline - & - & - & $1292^{(a)}$ & 9.2 & 0.027 & & & \\
\hline $1293(1)^{(b)}$ & 18.8 & 0.53 & - & - & - & - & - & \\
\hline - & - & - & 1302 & 6.1 & 0.018 & 1280 & 6.9 & 0.017 \\
\hline \multirow[t]{2}{*}{$1357(1)^{(b)}$} & 17.8 & 0.86 & 1336 & 210.7 & 0.612 & 1329 & 280.8 & 0.678 \\
\hline & & & & 19.5 & 57 & & & \\
\hline \multirow[t]{2}{*}{ 1374(1) } & 6.3 & 0.47 & $1373^{(a)}$ & 25.4 & 0.074 & & & \\
\hline & & & 13 & 9.7 & & & & \\
\hline - & _- & _- & $1387^{(a)}$ & 8.4 & 24 & & & \\
\hline $1429(2)^{(b)}$ & 16.4 & 0.5 & 1440 & 75.5 & 0.219 & 1422 & 101.8 & 0.246 \\
\hline & 8.3 & 0.4 & 14 & 20.3 & 0. & 1435 & 5.3 & 0.013 \\
\hline $1481(1)$ & 11.3 & 1 & 14 & 344.1 & 1.000 & 1460 & 414.2 & 1.00 \\
\hline- & - & - & $1485^{(a)}$ & 7.1 & 0.021 & & & \\
\hline - & - & - & $1520^{(a)}$ & 10.3 & 0.030 & & & \\
\hline- & - & - & $1529^{(a)}$ & 15.9 & 0.046 & & & \\
\hline - & - & - & 1558 & 88.3 & 0.257 & 1542 & 114.9 & 0.27 \\
\hline \multirow[t]{6}{*}{$1564(1)$} & 20 & 0.89 & 1562 & 85.6 & 0.249 & 1548 & 229.3 & 0.55 \\
\hline & & & $1562^{(a)}$ & 5.2 & 0.015 & & & \\
\hline & & & $1563^{(a)}$ & 75.1 & 0.218 & & & \\
\hline & & & $1564^{(a)}$ & 6.4 & 0.019 & & & \\
\hline & - & - & & 14 & 0.041 & & & \\
\hline & - & - & $1605^{(a)}$ & 18.2 & 0.053 & & & \\
\hline
\end{tabular}

Notes. Values in brackets denote uncertainties $(1 \sigma)$ in units of the last significant digit. ${ }^{(a)}$ Combination bands. ${ }^{(b)}$ Bands due to phen ${ }^{2+}$ contamination (including overlapping bands).

tropylium cations $\left(\mathrm{C}_{7} \mathrm{H}_{7}{ }^{+}\right)$, two isomers formed in dissociative ionization of toluene (Jusko et al. 2018a), but we did not observe any change in the peak intensity for any of the phen ${ }^{2+}$ bands. Instead, the intensity of the phen ${ }^{2+}$ bands decreased over time during the measurement campaign, leading us to conclude that they are due to contamination instead of isomerization. Relative depletion values on the isolated phen ${ }^{2+}$ bands at $786 \mathrm{~cm}^{-1}$ and $854 \mathrm{~cm}^{-1}$ indicate a contamination of $40-20 \%$, decreasing during the measurement campaign. The bottom panel of Fig. 4 shows the difference spectrum where the experimental (scaled) phen $^{2+}$ spectrum was subtracted from the $a^{2+h^{2+}}$ spectrum to 
account for this contamination. For this, we used an average $30 \%$ scaling factor over the whole spectral region, whereas the contamination varied from 40 to $20 \%$ over the course of the measurements, as outlined above. This leads to some remaining phen $^{2+}$ artifacts in the difference spectrum, for example, several bands between $1100-1200 \mathrm{~cm}^{-1}$ have not been completely removed, whereas several negative artifacts appear in the lower wavenumber range. Overall, however, this procedure allows for a better comparison to the calculated spectrum, as given in Table 3.

\section{Astrophysical implications and conclusions}

It is known that the charge state of the PAH plays an important role in the IR emission spectrum, especially in the 6-9 $\mu \mathrm{m}$ region where singly and multiply charged cations of PAH show strong bands (Langhoff 1996; Hudgins \& Allamandola 1999; Allamandola et al. 1999; Bauschlicher \& Bakes 2000). Witt et al. (2006) also proposed PAH dications as potential carriers of the extended red emission (ERE) observed in the reflection nebula NGC 7023 and the Red Rectangle nebula via fluorescence. In the ISM, PAHs can reach the doubly charged state in regions of high UV radiation density (e.g., in the PDR of the Orion Bar), through sequential absorption of two photons (Leach 1986). The second ionization energies of PAH cations are below $13.6 \mathrm{eV}$ (the ionization potential of atomic hydrogen), decreasing for larger species (Wenzel et al. 2020; Johnson 2019). In such regions, they play an important role in the chemistry, which in turn is affected by their stability. There has been some discussion in the past on the stability of PAH dications, since their energies are often higher than those of their singly charged fragments due to Coulomb repulsion (Leach 1996). At higher internal energies, $5 \mathrm{eV}$ above the second ionization energy, for example provided during the ionization process, fragmentation channels via covalent dissociation into a smaller dication fragment and a neutral also open up. We see this channel in the mass spectrum for anthracene electron impact ionization above energies of 23(2) eV with the appearance of a mass peak at $m / z=76$, which we interpret as the $\mathrm{C}_{12} \mathrm{H}_{8}$ fragment dication. However, often the dissociation is hindered by barriers in the potential energy surface along the dissociation coordinate, as has been shown in the case of the benzene dication (Rosi et al. 2004; Jašík et al. 2014). Earlier mass-spectrometric studies reported a yield of roughly $10 \%$ for PAH dications upon ionization of the neutral, with only marginal variations for different PAH sizes and ionization methods (e.g., EI and photo-ionization; Leach et al. 1989a,b; Zhen et al. 2017, 2016). We see a similar behaviour for the three PAH dications considered in this study. As exemplarily shown for the anthracene ions in Figs. 1 and 2, we reach dication to monocation ratios of up to $30 \%$ at high electron impact energies, and a dication yield of around $10 \%$ compared to all observed fragment plus parent ions. Evaluation of the abundance of PAH dications in the ISM, where they need to be produced by sequential photoionization competing with dissociation, chemical reactions, and recombination processes, requires detailed modelling of the $\mathrm{PAH}$ evolution. In particular, the comparably small PAH dications studied here are likely not stable in interstellar conditions (Montillaud et al. 2013; Zhen et al. 2015, 2016). It would therefore be interesting to extend the vibrational studies presented here to the class of larger, astronomically more relevant PAH dications.

To date, vibrational spectral information for PAH dications comes mainly from theoretical calculations, and we are aware of only one experimental IR study by Zhen et al. (2018).
Malloci et al. did a comparative theoretical study on IR properties of 40 different PAH neutrals, monocations, and dications using DFT and TD-DFT theoretical techniques. Based on these calculations, most of the dications were predicted to have a singlet ground state including the dications presented in this work and our recorded spectra support this finding. Another notable characteristic in the recorded IR spectra of all three dications is the presence of several intense combination bands, especially in the $5-10 \mu \mathrm{m}$ region. Here, anharmonic calculations proved to be crucial in assigning these combination bands. The presence of combination bands has an effect on the fraction of the total integrated intensity (InI) in the different spectral ranges $2.5-3.5,5-10,10-15$, and $>15 \mu \mathrm{m}$. Differences in the relative integrated intensities in these spectral ranges serve as identifiers for the charge state of PAHs in the ISM, as has been discussed in detail previously (Bauschlicher \& Bakes 2000; Malloci et al. 2007a). Whereas drastic changes in the integrated intensities were observed between neutral and cationic species, there were much fewer predicted variations between the singly and doubly charged PAHs. The question arises if the appearance of strong combination bands changes this picture. A comparison is made in Appendix $\mathrm{C}$ showing the effect of including anharmonicity in the calculations on the integrated intensities of singly and doubly charged PAHs considered in this work. As the choice of the basis set in theoretical calculations of vibrational spectra has been discussed previously (Andersson \& Uvdal 2005; Bauschlicher \& Langhoff 1997), we first compared the InI for the two functionals B3LYP/6-311+G(d,p) (this work) and B3LYP/4-31G (Malloci et al. 2007a), using the harmonic approximation, see Fig. C.1. We can see that there is no significant effect on InI for either the singly or doubly charged species. However, when performing anharmonic calculations (with the B3LYP/6-311+G(d,p) functional), the InI in the 2.5-5 $\mu \mathrm{m}$ region is 5-7\% higher for both singly and doubly charged PAHs compared to harmonic calculations using the same functional. In contrast, in the $5-10 \mu \mathrm{m}$ region, InI predicted by anharmonic calculations is lower compared to harmonic calculations by $28 \%$ for naph ${ }^{+1}, 15 \%$ for anth $^{+1}$, and phen ${ }^{+1}$, but only 2-5\% for doubly charged PAHs. In the spectral regions $>15 \mu \mathrm{m}$ and $10-15 \mu \mathrm{m}$, both singly and doubly charged anth and phen show negligible $(<1 \%)$ changes in InI, whereas naph shows $7 \%$ and $12 \%$ higher InI predicted with anharmonic compared to harmonic calculations. Taking into account the fact that the absolute intensities of the fundamental bands are generally predicted lower in anharmonic calculations than harmonic (see Tables 2, 3), about $45 \%$ and $25 \%$ of the absolute integrated intensity arises entirely from combination bands of singly and doubly charged phen and anth, respectively.

The significant differences observed in the relative integrated intensities in each of the spectral regions and the presence of combination bands suggest that anharmonic calculations are necessary to predict accurate vibrational spectra for the family of comparatively small PAH cations as discussed here and also in previous work (Maltseva et al. 2015; Lemmens et al. 2019). Thus, including these anharmonic effects has consequences on the interpretation of relative UIR band intensities observed in the ISM.

Here, we have shown that cryogenic IRPD experiments using $\mathrm{Ne}$-tagging prove to be a very powerful method to obtain vibrational spectra of PAH dications by providing narrow features and intensities being more comparable to the linear absorption cross section. This makes it a very effective tool to benchmark theoretical calculations. In order to validate our finding that anharmonic effects significantly influence the IR band positions and intensities of PAH monocations and dications, future IRPD 
experiments targeting larger and structurally different classes of PAH cations should be conducted. As discussed above, the double ionization process competes with fragmentation channels. One of the main fragmentation channels observed for PAHs is the loss of acetylene, $\mathrm{C}_{2} \mathrm{H}_{2}$ (Johansson et al. 2011; Simon et al. 2017; Ling \& Lifshitz 1998), as we have also observed in this study (see Fig. 2). Elucidating the structure of these $\left(\mathrm{C}_{2} \mathrm{H}_{2}\right)$-loss fragment ions, as has been previously done for the case of naphthalene (Bouwman et al. 2016), is another interesting application for the narrow-linewidth IRPD action spectroscopy that we have presented here.

Acknowledgements. This project is funded by the Marie Skłodowska Curie Actions (MSCA) Innovative Training Networks (ITN) H2020-MSCA-ITN 2016 (EUROPAH project, G.A. 722346). We are grateful for the experimental support provided by the FELIX team and acknowledge the Nederlandse Organisatie voor Wetenschappelijk Onderzoek (NWO) for the support of the FELIX Laboratory. We also thank NWO Exact and Natural Sciences for the use of supercomputer facilities (Grant no. 2019.062). We thank the Cologne Laboratory Astrophysics group for providing the FELion ion trap instrument for the current experiments and the Cologne Center for Terahertz Spectroscopy (core facility, DFG grant SCHL 341/15-1) for supporting its operation. We would like to thank the anonymous referee for valuable comments which helped to improve the manuscript.

\section{References}

Allamandola, L. J., Tielens, A. G. G. M., \& Barker, J. R. 1985, ApJ, 290, L25 Allamandola, L. J., Tielens, A. G. G. M., \& Barker, J. R. 1989, ApJS, 71, 733

Allamandola, L. J., Hudgins, D. M., \& Sandford, S. A. 1999, ApJ, 511, L115

Alvaro Galué, H., Pirali, O., \& Oomens, J. 2010, A\&A, 517, A15

Andersson, M. P., \& Uvdal, P. 2005, J. Phys. Chem. A, 109, 2937

Asmis, K. R., Brümmer, M., Kaposta, C., et al. 2002, Phys. Chem. Chem. Phys. 4, 1101

Bakes, E., Tielens, A., Bauschlicher Jr, C. W., Hudgins, D. M., \& Allamandola, L. J. 2001a, ApJ, 560, 261

Bakes, E. L. O., Tielens, A. G. G. M., \& Bauschlicher, C. 2001b, ApJ, 556, 501

Bakker, J. M., Redlich, B., van der Meer, A. F. G., \& Oomens, J. 2011, ApJ, 741, 74

Bauschlicher, C. W., \& Langhoff, S. R. 1997, Spectrochim. Acta A Mol. Biomol Spectrosc., 53, 1225

Bauschlicher, C., \& Bakes, E. 2000, Chem. Phys., 262, 285

Bouwman, J., de Haas, A. J., \& Oomens, J. 2016, Chem. Commun., 52, 2636

Bouwman, J., Boersma, C., Bulak, M., et al. 2020, A\&A, 636, A57

Brünken, S., Lipparini, F., Stoffels, A., et al. 2019, J. Phys. Chem. A, 123, 8053

Chen, T. 2018, ApJS, 238, 18

Cohen, M., Allamandola, L., Tielens, A. G. G. M., et al. 1986, ApJ, 302, 737

Dartois, E., \& D'Hendecourt, L. 1997, A\&A, 323, 534

Frisch, M. J., Trucks, G. W., Schlegel, H. B., et al. 2016, Gaussian-16 Revision C.01 (Wallingford CT: Gaussian Inc.)

Galliano, F., Madden, S. C., Tielens, A. G. G. M., Peeters, E., \& Jones, A. P. 2008, ApJ, 679, 310

Gao, J., Berden, G., \& Oomens, J. 2014, ApJ, 787, 170

Gerlich, D. 1992, Inhomogeneous RF fields: A versatile Tool for the Study of processes with Slow Ions, Vol. LXXXII (New York: Wiley), 1-176

Gerlich, D., Jašík, J., Strelnikov, D. V., \& Roithová, J. 2018, ApJ, 864, 62

Günther, A., Nieto, P., Müller, D., et al. 2017, J. Mol. Spectrosc., 332, 8

Holm, A. I. S., Johansson, H. A. B., Cederquist, H., \& Zettergren, H. 2011, J Chem. Phys., 134, 044301

Hony, S., Van Kerckhoven, C., Peeters, E., et al. 2001, A\&A, 370, 1030
Hudgins, D. M., \& Allamandola, L. J. 1999, ApJ, 513, L69

Hudgins, D., Allamandola, L., \& Sandford, S. 1997, Adv. Space Res., 19, 999

Jašík, J., Gerlich, D., \& Roithová, J. 2014, J. Am. Chem. Soc., 136, 2960

Johnson, D. R. 2019, NIST Computational Chemistry Comparison and Benchmark Database, nIST Standard Reference Database Number 101, Release 20 , http://cccbdb.nist.gov/

Johansson, H. A. B., Zettergren, H., Holm, A. I. S., et al. 2011, J. Chem. Phys., 135,084304

Jourdain de Muizon, M., D’Hendecourt, L. B., \& Geballe, T. R. 1990, A\&A, 235,367

Jusko, P., Simon, A., Banhatti, S., Brünken, S., \& Joblin, C. 2018a, ChemPhysChem, 19, 3173

Jusko, P., Simon, A., Wenzel, G., et al. 2018b, Chem. Phys. Lett., 698, 206

Jusko, P., Brünken, S., Asvany, O., et al. 2019, Faraday Discuss., 217, 172

Langhoff, S. R. 1996, J. Phys. Chem. A, 100, 2819

Leach, S. 1986, J. Electron Spectr. Rel. Phenomena, 41, 427

Leach, S. 1996, Zeitsch. Physik. Chem., 195, 15

Leach, S., Eland, J., \& Price, S. 1989a, J. Phys. Chem. A, 93, 7575

Leach, S., Eland, J., \& Price, S. 1989b, J. Phys. Chem. A, 93, 7583

Leger, A., \& Puget, J. L. 1984, A\&A, 137, L5

Lemmens, A. K., Rap, D. B., Thunnissen, J. M. M., et al. 2019, A\&A, 628, A130

Ling, Y., \& Lifshitz, C. 1998, J. Phys. Chem. A, 102, 708

Lorenz, U., Solcà, N., Lemaire, J., Maître, P., \& Dopfer, O. 2007, Angew. Chem. Int. Ed., 46, 6714

Mackie, C. J., Candian, A., Huang, X., et al. 2015, J. Chem. Phys., 143, 224314

Mackie, C. J., Candian, A., Huang, X., et al. 2018, Phys. Chem. Chem. Phys., 20, 1189

Malloci, G., Joblin, C., \& Mulas, G. 2007a, Chem. Phys., 332, 353

Malloci, G., Mulas, G., Cappellini, G., \& Joblin, C. 2007b, Chem. Phys., 340, 43

Maltseva, E., Petrignani, A., Candian, A., et al. 2015, ApJ, 814, 23

Montillaud, J., Joblin, C., \& Toublanc, D. 2013, A\&A, 552, A15

Oepts, D., van der Meer, A. F. G., \& van Amersfoort, P. W. 1995, Infrared Phys. Technol., 36, 297

Oomens, J., van Roij, A. J. A., Meijer, G., \& von Helden, G. 2000, ApJ, 542, 404

Oomens, J., Sartakov, B. G., Tielens, A. G. G. M., Meijer, G., \& von Helden, G. 2001, ApJ, 560, L99

Panchagnula, S., Bouwman, J., Rap, D. B., et al. 2020, Phys. Chem. Chem. Phys., 22,21651

Piccardo, M., Bloino, J., \& Barone, V. 2015, Int. J. Quant. Chem., 115, 948

Piest, H., von Helden, G., \& Meijer, G. 1999, ApJ, 520, L75

Piest, H., Oomens, J., Bakker, J., von Helden, G., \& Meijer, G. 2001, Spectrochim. Acta A Mol. Biomol. Spectrosc., 57, 717

Ricks, A. M., Douberly, G. E., \& Duncan, M. A. 2009, ApJ, 702, 301

Rosi, M., Bauschlicher Jr, C. W., \& Bakes, E. 2004, ApJ, 609, 1192

Sellgren, K. 1984, ApJ, 277, 623

Sellgren, K., Allamandola, L. J., Bregman, J. D., Werner, M. W., \& Wooden, D. H. 1985, ApJ, 299, 416

Simon, A., Rapacioli, M., Rouaut, G., \& Gadéa, F. 2017, Phil. Trans. Roy. Soc. A, 375, 20160195

Solano, E. A., \& Mayer, P. M. 2015, J. Chem. Phys., 143, 104305

Tielens, A. 2008, ARA\&A, 46, 289

van der Burgt, P. J. M., Dunne, M., \& Gradziel, M. L. 2018, Eur. Phys. J. D, 72, 31

van der Burgt, P. J. M., Dunne, M., \& Gradziel, M. L. 2019, J. Phys. Conf. Ser., 1289,012008

Wenzel, G., Joblin, C., Giuliani, A. A., et al. 2020, A\&A, 641, A98

Witt, A. N., Gordon, K. D., Vijh, U. P., et al. 2006, ApJ, 636, 303

Zhen, J., Castellanos, P., Paardekooper, D. M., et al. 2015, ApJ, 804, L7

Zhen, J., Castillo, S. R., Joblin, C., et al. 2016, ApJ, 822, 113

Zhen, J., Castellanos, P., Bouwman, J., Linnartz, H., \& Tielens, A. G. G. M. 2017, ApJ, 836, 28

Zhen, J., Candian, A., Castellanos, P., et al. 2018, ApJ, 854, 27 


\section{Appendix A: Mass spectrum showing doubly charged anthracene tagged with $\mathrm{Ne}$}

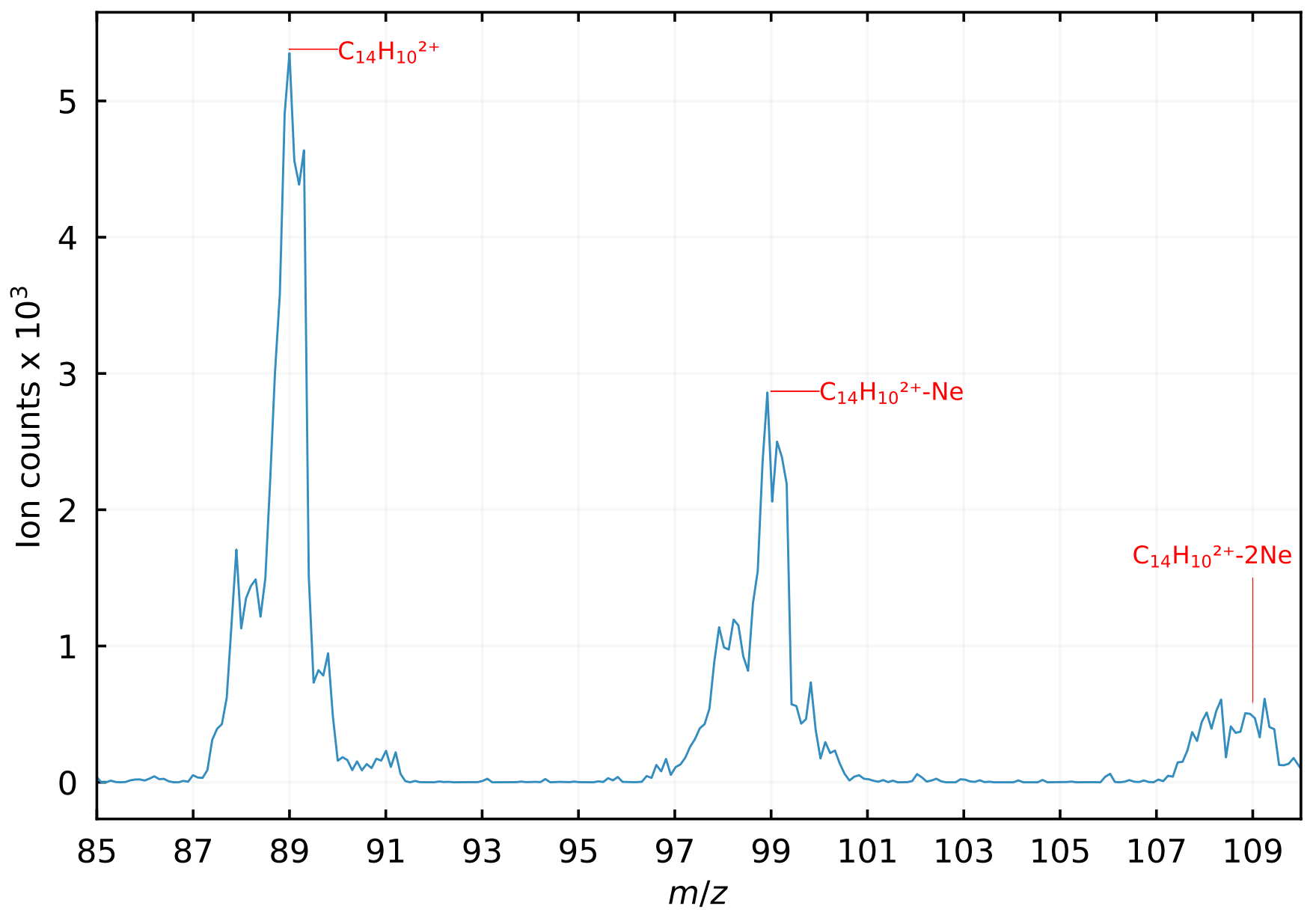

Fig. A.1. Mass spectrum showing anth ${ }^{2+}\left(\mathrm{C}_{14} \mathrm{H}_{10}{ }^{2+}, m / z=89\right)$ with one $\left(\mathrm{C}_{14} \mathrm{H}_{10}{ }^{2+}-\mathrm{Ne}, m / z=99\right)$ and two $\left(\mathrm{C}_{14} \mathrm{H}_{10}{ }^{2+}-\mathrm{Ne}_{2}, m / z=109\right)$ attached neon atoms. Additional mass peaks appearing with $\Delta m / z=0.5$ can be seen in the spectrum for the bare ion and Ne-dication complexes. They belong to dehydrogenated phen ${ }^{2+}$ (at lower mass) and ${ }^{13} \mathrm{C}$ substituted or hydrogenated phen ${ }^{2+}$ (higher masses), respectively, see also van der Burgt et al. (2018). The mass resolution of the second quadrupole mass filter is better than $m / z=0.5$, which allowed us to mass select only the singly tagged target complex to record the IRPD spectra. 
Appendix B: Comparison of vibrational spectra of bare and Ne-tagged $\mathrm{Naph}^{2+}$

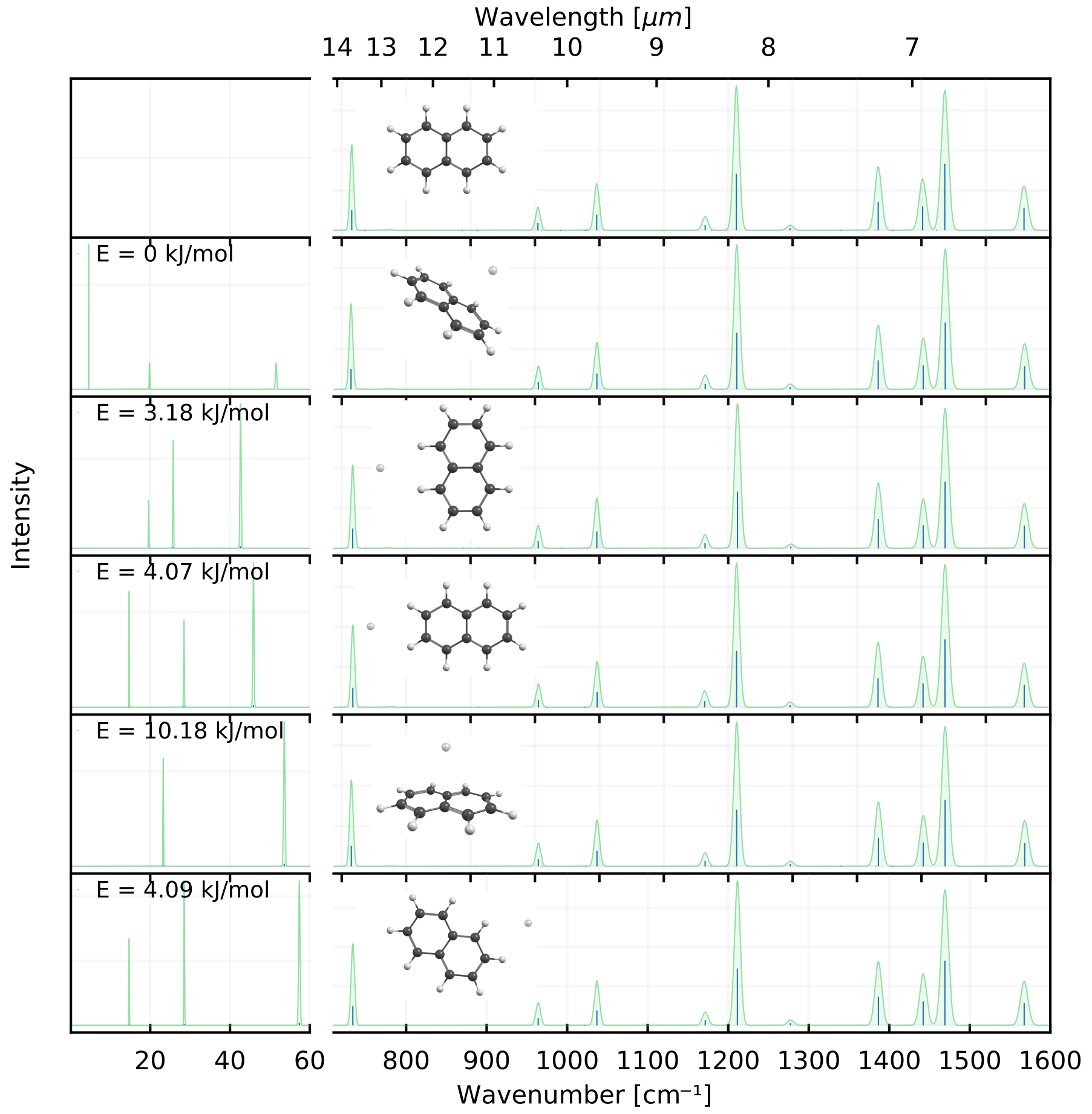

Fig. B.1. Vibrational spectra of doubly charged bare naphthalene and neon-tagged naphthalene calculated using DFT within the harmonic approximation at the wB97XD/cc-pVTZ level. The respective energies of the five lowest conformers relative to the lowest energy conformer are stated. The frequency shifts between the different neon isomers and the bare ion are negligible. The three bands observed in the $0-60 \mathrm{~cm}^{-1}$ region are the bending and stretching vibrations involving the neon atom where we observe large shifts in frequencies among its isomers. 
S. Banhatti et al.: Infrared action spectroscopy of doubly charged PAHs and their contribution to the aromatic infrared bands

Appendix C: Comparison between anharmonic and harmonic calculations
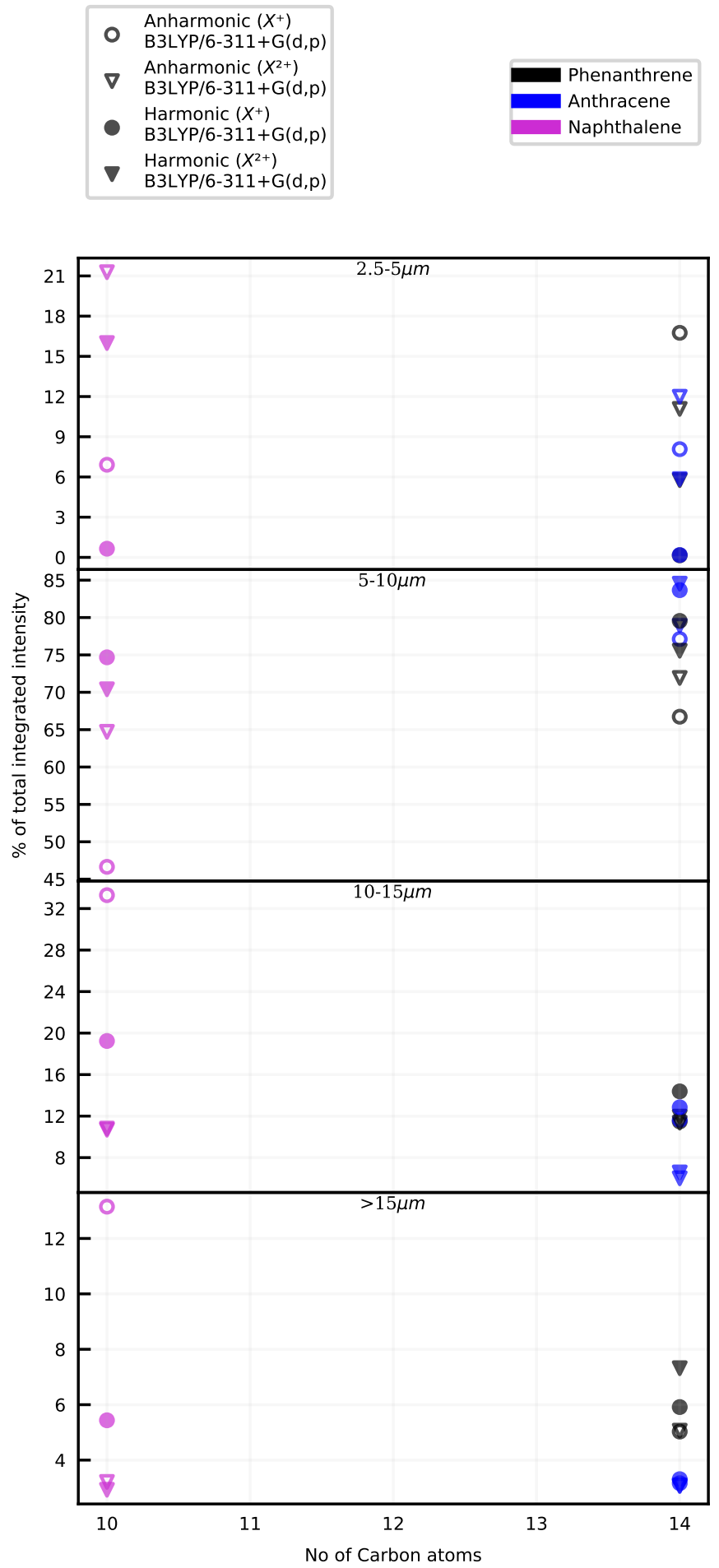

Harmonic $\left(X^{+}\right)$ $B 3 L Y P / 6-311+G(d, p)$

Harmonic $\left(X^{2+}\right)$

B3LYP/6-311+G(d,p)

Harmonic $\left(X^{+}\right)$

B3LYP/4-31G

Harmonic $\left(X^{2+}\right)$

B3LYP/4-31G

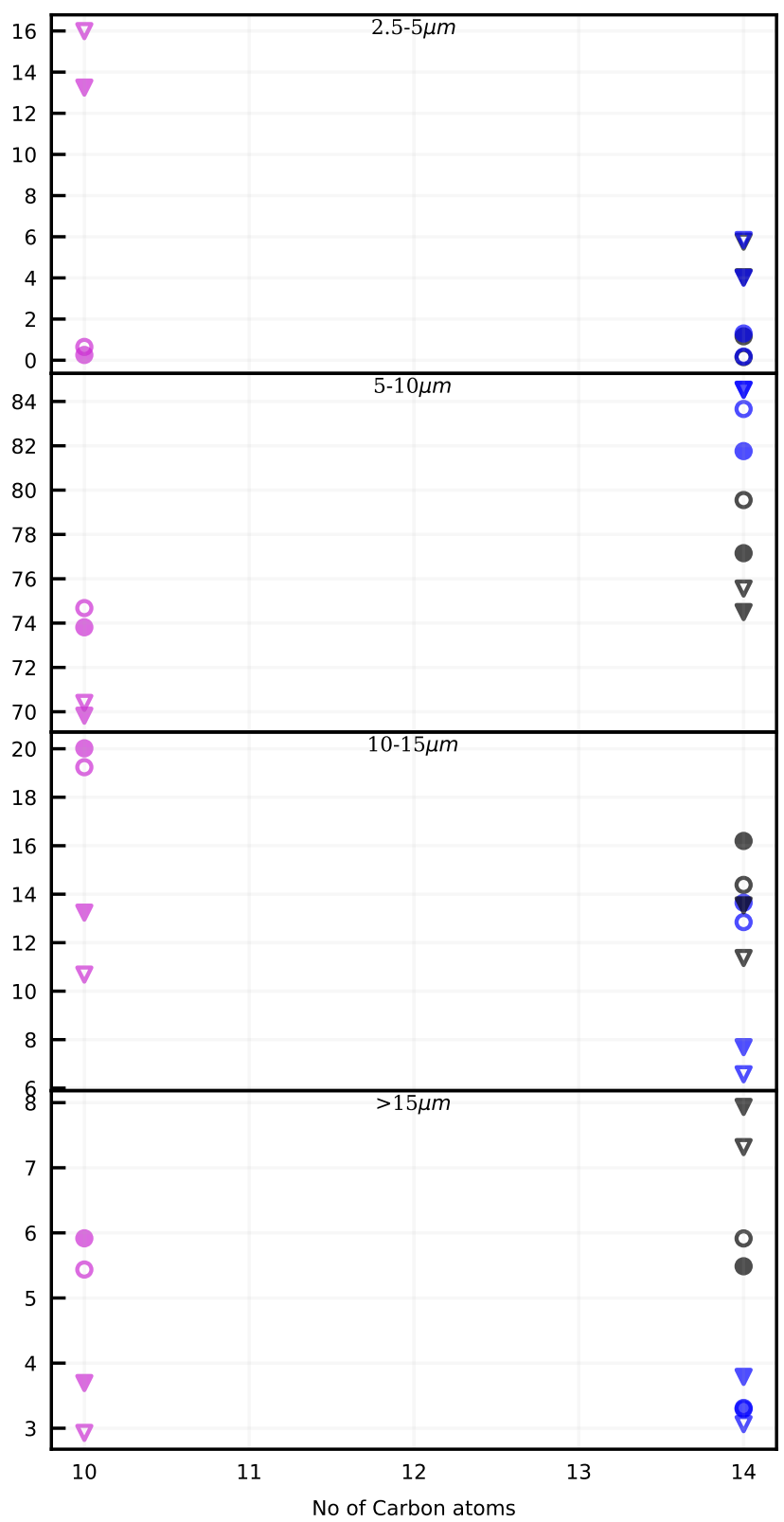

Fig. C.1. Integrated intensities in different regions of the mid IR spectra. Left panel: comparison made between harmonic calculations using the B3LYP/6-311+G(d,p) and B3LYP/4-31G functionals. Right panel: comparison made between anharmonic and harmonic calculations using the B3LYP/6-311+G(d,p) functional. 
Table C.1. DFT computed harmonic (scaled by 0.9679) fundamental mode positions $v_{\text {vib }}\left(\mathrm{cm}^{-1}\right)$ and intensities $I\left(\mathrm{kmmol}^{-1}\right)>5 \mathrm{~km} \mathrm{~mol}^{-1}$ of triplet electronic ground state for naph ${ }^{2+}$, anth ${ }^{2+}$, and $\mathrm{phen}^{2+}$.

\begin{tabular}{|c|c|c|c|c|c|}
\hline \multicolumn{2}{|c|}{ naph $^{2+}$} & \multicolumn{2}{|c|}{$\mathrm{anth}^{2+}$} & \multicolumn{2}{|c|}{ phen $^{2+}$} \\
\hline$v_{\mathrm{vib}}$ & $I$ & $v_{\text {vib }}$ & $I$ & $v_{\text {vib }}$ & $I$ \\
\hline 137 & 5.1 & 252 & 28.2 & 346 & 12 \\
\hline 407 & 17.6 & 410 & 26.7 & 401 & 8.1 \\
\hline 755 & 97.5 & 548 & 23.9 & 712 & 91.8 \\
\hline 922 & 216.9 & 715 & 87.6 & 787 & 22 \\
\hline 976 & 9.6 & 741 & 113.6 & 808 & 47.1 \\
\hline 1064 & 57.4 & 781 & 153.8 & 849 & 13.7 \\
\hline 1138 & 32.6 & 864 & 50.9 & 927 & 119.9 \\
\hline 1186 & 15.2 & 880 & 31.9 & 991 & 9.6 \\
\hline 1189 & 11.8 & 902 & 296.9 & 1019 & 20.6 \\
\hline 1365 & 19.7 & 943 & 16.5 & 1046 & 9.4 \\
\hline 1401 & 269.2 & 1133 & 13.1 & 1085 & 10.5 \\
\hline 1416 & 250.9 & 1294 & 210.9 & 1114 & 18 \\
\hline 3082 & 8 & 1349 & 386.7 & 1164 & 45 \\
\hline 3089 & 94.7 & 1373 & 81.8 & 1170 & 168.5 \\
\hline \multirow[t]{15}{*}{3093} & 91.6 & 1376 & 269.5 & 1209 & 6 \\
\hline & & 1393 & 42.5 & 1302 & 7.8 \\
\hline & & 2982 & 7.5 & 1310 & 24.1 \\
\hline & & 2989 & 6.1 & 1364 & 8.8 \\
\hline & & 2998 & 41.8 & 1381 & 101.4 \\
\hline & & 3003 & 48.2 & 1385 & 34.2 \\
\hline & & & & 1422 & 59.4 \\
\hline & & & & 1439 & 13.8 \\
\hline & & & & 1441 & 87.2 \\
\hline & & & & 1490 & 44.3 \\
\hline & & & & 1509 & 25.6 \\
\hline & & & & 2996 & 32.2 \\
\hline & & & & 3002 & 49 \\
\hline & & & & 3009 & 11.2 \\
\hline & & & & 3022 & 10.4 \\
\hline
\end{tabular}

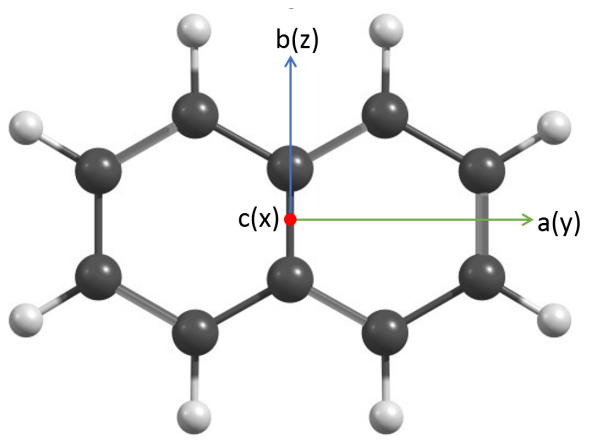

Fig. C.2. Naphthalene dication showing the orientation of the molecular axes used in the calculations ( $I I^{r}$ representation), defining the symmetry labels given in Table 1. Inertia moments: Iy $<\mathrm{Iz}<\mathrm{Ix}$. 